\title{
Scrambling and Gate-Induced Fluctuations in Realistic Quantum Dots
}

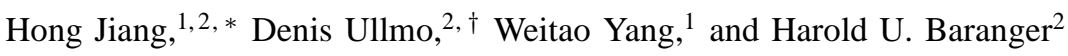 \\ ${ }^{1}$ Department of Chemistry, Duke University, Durham, North Carolina 27708-0354 \\ ${ }^{2}$ Department of Physics, Duke University, Durham, North Carolina 27708-0305
}

(Dated: November 20, 2018)

\begin{abstract}
We evaluate the magnitude of two important mesoscopic effects using a realistic model of typical quantum dots. "Scrambling" and "gate effect" are defined as the change in the single-particle spectrum due to added electrons or gate-induced shape deformation, respectively. These two effects are investigated systematically in both the self-consistent Kohn-Sham (KS) theory and a Fermi liquid-like Strutinsky approach. We find that the genuine scrambling effect is small because the potential here is smooth. In the KS theory, a key point is the implicit inclusion of residual interactions in the spectrum; these dominate and make scrambling appear larger. Finally, the gate effect is comparable in the two cases and, while small, is able to cause gate-induced spin transitions.

PACS numbers: 73.23.Hk, 73.40.Gk, 73.63.Kv
\end{abstract}

\section{INTRODUCTION}

An important way to characterize quantum dots (QDs),, 1.2 .3$ the simplest artificial nano-structure with electrons quantized in all three dimensions, is by the parametric evolution of their properties. The most common external parameter is magnetic field because of its flexibility of tuning, 1 but other parameters are also used. Here we are concerned with the effect of changing the electron number $N$ or the external gate voltage $U_{\mathrm{g}}$, referred to as the scrambling and gate effects, respectively, in Coulomb blockade (CB) experiments 4.5.6.7.8.9.10 The most striking feature of the $\mathrm{CB}$ regime is sharp peaks in the conductance through the quantum dot as a function of gate voltage. As shown in Fig. 1 at each conductance peak, the number of electrons residing in the dot changes by one; across a peak spacing, the gate voltage changes to bring another electron into the dot, and deforms the confining potential in the meantime. The scrambling and gate-induced shape deformation effects were both introduced ${ }^{11,12}$ in connection with experiments on the spacing between $\mathrm{CB}$ conductance peaks,, ,6.7.8.9 and have also been used to interpret CB peak height correlations $\underline{\underline{10}}$

The scrambling and gate effects can both be quantified through the variation in the single-particle spectrum of the system, $\left\{\varepsilon_{i}\right\}$. Since electron-electron interactions are important for quantum dots in the Coulomb blockade regime, one must clearly consider the effect of such interactions on the single-particle spectrum. Here we evaluate the scrambling and gate effects using both density functional theory and ThomasFermi calculations for realistic geometries of quantum dots. We address two main issues:

First, while the magnitude of the scrambling and gate effects has been estimated for hard-wall quantum dots coupled to a large gate (one which deforms the entire dot) ${ }^{13.14}$ experimental quantum dots have, of course, smooth confining potentials, and are typically deformed with a narrow "plunger" gate. We evaluate these experimental features using our realistic model of quantum dots, showing that they influence the magnitude of the scrambling and gate effects strongly.

Second, what "single-particle spectrum" should one use in evaluating the scrambling and gate effects? Roughly there are two types of single-particle spectra that can be defined in an interacting system. The first is a spectrum from a selfconsistent mean field theory such as Hartree-Fock (HF) ${ }^{15}$ or Kohn-Sham spin-density functional theory (KS-SDFT) 16 The second is the spectrum of a reference Hamiltonian which contains the interactions only at a smooth (classical-like) level. The most natural choice is the eigen-spectrum of the effective potential calculated from Thomas-Fermi (TF) theory; this

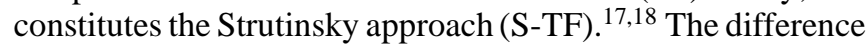
between these two types of spectra is familiar, for instance, from discussions of the meaning of the eigenvalues in the self-consistent approach: Recall that the self-consistent eigenvalue is related to the energy for removing an electron from that level and that a sum over such eigenvalues double counts the interaction energy among those electrons, $\frac{16,19}{19}$ neither of which is true for the eigen-spectrum in the reference Hamiltonian approach.

The meaning and magnitude of the scrambling and gate effects depend on which type of single-particle spectrum is used. We emphasize that this is not a question of which approach is the more accurate, but rather of what part of the fluctuations of the total energy is assigned to these effects. For instance, when using a reference potential as in S-TF, the fluctuations as a parameter changes associated with interactions are separated into two distinct parts. The first comes from changes in the single-particle energies as the smooth Thomas-Fermi potential varies - we consider these the "gen-

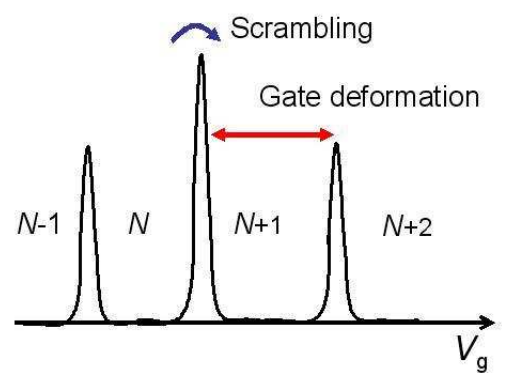

FIG. 1: (Color online) Illustration of scrambling and gate effects in Coulomb blockade conductance fluctuations. 
uine" scrambling and gate effects. The second contribution involves the screened Coulomb interaction between mesoscopic density fluctuations. This "residual interaction" term corresponds to the weak interaction between Landau quasiparticles in a Fermi liquid picture. In a self-consistent approach such as KS-SDFT, they appear in the self-consistent energies $\varepsilon_{i}^{\mathrm{KS}}(N)$; thus parametric evolution of the KS levels involves both genuine scrambling and residual interactions.

The paper is organized as followings. In the next section, we outline briefly the two theoretical approaches, i.e. the Kohn-Sham method and Fermi-liquid like Strutinsky approximation scheme. In Section III, we describe the 2D realistic quantum dot model used for the study the scrambling and gate effects. The main results of this study are presented and analyzed in Section IV.

\section{THEORY}

In both the KS-SDFT and S-TF approaches, for a system with $N$ electrons and total spin $S_{\mathrm{z}}$, one solves a Schrödinger equation with a spin-dependent potential,

$$
\begin{gathered}
\left\{-\frac{1}{2} \nabla^{2}+U^{\sigma}(\mathbf{r})\right\} \psi_{i}^{\sigma}(\mathbf{r})=\varepsilon_{i}^{\sigma} \psi_{i}^{\sigma}(\mathbf{r}) \\
U^{\sigma}(\mathbf{r})=U_{\mathrm{ext}}(\mathbf{r})+U_{H}(\mathbf{r} ;[n])+U_{\mathrm{xc}}^{\sigma}\left(\mathbf{r} ;\left[n^{\alpha}, n^{\beta}\right]\right)
\end{gathered}
$$

where the total potential is the sum of the external, Hartree, and exchange-correlation contributions, and $\sigma=\alpha, \beta$ denotes spin-up and down, respectively. In KS-SDFT, 16 one knows that the potentials are functionals of the spin densities $n^{\sigma}(\mathbf{r})=\sum_{i}^{N^{\sigma}}\left|\psi_{i}^{\sigma}(\mathbf{r})\right|^{2}$, which are solved self-consistently under the constraint $\int n^{\sigma}(\mathbf{r}) d \mathbf{r}=N^{\sigma}$ with $N^{\alpha}=\left(N+2 S_{\mathrm{z}}\right) / 2$ and $N^{\beta}=\left(N-2 S_{\mathrm{z}}\right) / 2$. In analogy to the Koopmans's theorem in Hartree-Fock theory, it has been proved that the highest occupied Kohn-Sham orbital energy is identical to the chemical potential if the exact exchange-correlation potential is used ${ }^{20}$ This association provides a physical meaning to the self-consisent eigenvalues which can be contrasted with that of the reference Hamiltonian eigenvalues.

In the S-TF approach, the basic idea is to start from a smooth semiclassical approximation, i.e. the ThomasFermi theory, and introduce quantum interference by considering, first, single-particle corrections and, then, the effect of screened interactions between the oscillating part of the electron density. In this case, then, one uses the TF potential in Eq. (11). From the resulting $\varepsilon_{i}^{\sigma}$ and $\psi_{i}^{\sigma}$ one obtains an approximation to the KS-SDFT total energy valid up through secondorder in the oscillating density, $n_{\mathrm{OSc}}^{\sigma}(\mathbf{r}) \equiv n^{\sigma}(\mathbf{r})-n_{\mathrm{TF}}(\mathbf{r}){ }^{17.18}$ In this approximation, the relation between the KS-SDFT and Thomas Fermi single-particle levels can be expressed as 18

$$
\varepsilon_{i, \sigma}^{\mathrm{KS}} \approx \varepsilon_{i, \sigma}^{\mathrm{TF}}+\delta \varepsilon_{i, \sigma}^{\mathrm{RI}}
$$

where the residual interaction terms

$$
\delta \varepsilon_{i, \sigma}^{\mathrm{RI}}=\sum_{\sigma^{\prime}} \int d \mathbf{r} d \mathbf{r}^{\prime}\left|\psi_{i}^{\sigma}(\mathbf{r})\right|^{2} V_{\mathrm{scr}}^{\sigma, \sigma^{\prime}}\left(\mathbf{r}, \mathbf{r}^{\prime}\right) n_{\mathrm{osc}}^{\sigma^{\prime}}\left(\mathbf{r}^{\prime}\right)
$$

corresponds to the interaction of $\psi_{i}^{\sigma}$ with the density ripples due to interferences. The screened interaction potential $V_{\mathrm{scr}}^{\sigma, \sigma^{\prime}}\left(\mathbf{r}, \mathbf{r}^{\prime}\right)$ is expressed explicitly in terms of the functional derivatives of $U^{\sigma}\left(\mathbf{r} ;\left[n^{\alpha}\right]\right) ! \frac{18}{1}$ When for instance an electron is added into the quantum dot, both the genuine scrambling effect (i.e. the variations of the Thomas Fermi levels) and the residual interaction terms Eq. (4) will affect the KS-spectrum.

In this study we evaluate the scrambling and gate effects in both KS and S-TF approaches. For the S-TF case, the spindependence of the potential has little effect on its spectrum other than a constant shift, so we use the spin-independent Thomas-Fermi potential. For KS, we first solve the full spindependent KS equations, but calculate the scrambling and gate effects only from $\alpha$ orbital energies. Only minimal spin states, $S_{\mathrm{z}}=0$ for even $N$ and $S_{\mathrm{z}}=1 / 2$ for odd $N$, are considered. The spin indices will therefore be dropped in the remainder of the paper. The numerical methods that are used to solve $\mathrm{KS}$ and TF equations are described in details in Refs 2122 , respectively.

\section{MODEL SYSTEM}

The model system we use for investigating gate and scrambling effects is a realistic 2D lateral quantum dot: ${ }^{23}$ The electrons are at the heterointerface a distance $z_{d}$ below the surface of the heterostructure. For the electrostatic potential, we use the mid-gap pinning model for the boundary condition at GaAs free surface: $24,25,26$ We impose Dirichlet boundary conditions on the top surface and Neumann conditions in infinity in all other directions $\stackrel{23,27}{\stackrel{20}{2}}$ allowing the external potential to be calculated from

$$
U_{\text {ext }}(\mathbf{r})=\frac{1}{2 \pi} \int d \mathbf{r}^{\prime} U_{\mathrm{g}}\left(\mathbf{r}^{\prime}\right) \frac{z_{\mathrm{d}}}{\left(\left|\mathbf{r}-\mathbf{r}^{\prime}\right|^{2}+z_{\mathrm{d}}^{2}\right)^{3 / 2}}+U_{\mathrm{QW}}\left(z_{\mathrm{d}}\right),
$$

where $U_{\mathrm{g}}(\mathbf{r})$ is the electrostatic potential on the top gate surface, ${ }^{26}$ and $U_{\mathrm{QW}}(z)$ is the confining potential in the growth direction due to the quantum well structure from which the quantum dot is fabricated. In addition, the Hartree potential has an image term due to the coupling with the top surface,, 24.27

$$
U_{\mathrm{H}}(\mathbf{r} ;[n])=\int d \mathbf{r}^{\prime} n\left(\mathbf{r}^{\prime}\right)\left[\frac{1}{\left|\mathbf{r}-\mathbf{r}^{\prime}\right|}-\frac{1}{\left(\left|\mathbf{r}-\mathbf{r}^{\prime}\right|^{2}+4 z_{\mathrm{d}}^{2}\right)^{1 / 2}}\right] .
$$

For a complete description of our treatment of the electrostatic potential see Ref. 23.

The shape of the top confining gate used here is shown in Fig 2 it is designed to model typical irregular dots investigated experimentally 6 Negative voltages are imposed on the top $\left(U_{\mathrm{t}}\right)$, bottom $\left(U_{\mathrm{b}}\right)$, and plunger $\left(U_{\mathrm{p}}\right)$ electrode gates; the electron number in the dot is controlled by $U_{\mathrm{p}}$. The singleparticle dynamics of the system is expected to be chaotic, which is confirmed by the agreement between the nearestneighboring spacing distribution of the single-particle levels and the Wigner surmise distribution ${ }^{28}$

We now introduce quantities to characterize the scrambling 


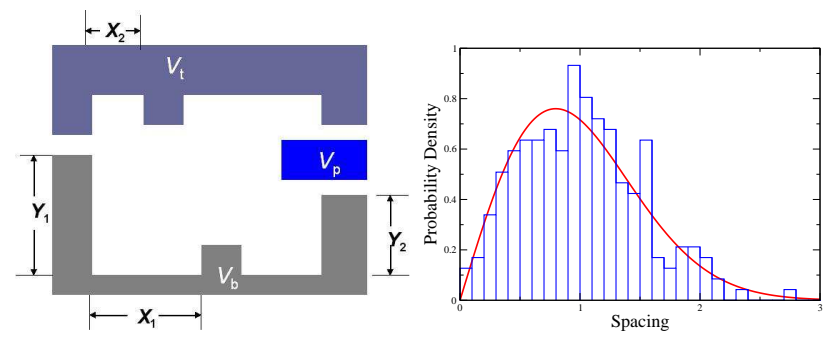

FIG. 2: (Color online) Left panel: Schematic of the shape of the top confining gate used in this study. Negative voltages are imposed on the shaded region, depleting the electrons underneath so that the motion of electrons is confined to a small region. To obtain enough statistics, 24 different irregular confining shapes are generated by taking different values of $X_{1}, X_{2}, Y_{1}$ and $Y_{2}$. Right panel: The nearest neighbor spacing distribution of the single-particle levels calculated in the Thomas-Fermi effective potential (histogram) compared to the Wigner surmise distribution (line).

and gate effects. For the scrambling effect, we define

$$
\delta \varepsilon_{i}(\delta N) \equiv \varepsilon_{i}\left(N^{(0)}+\delta N\right)-\varepsilon_{i}\left(N^{(0)}\right) .
$$

where $\varepsilon_{i}(N)$ is the $i^{\text {th }}$ single-particle energy in either the TF or KS effective potential with $N$ electrons. Scrambling is quantified by the magnitude of fluctuations in $\delta \varepsilon_{i}(\delta N)$ :

$$
\sigma_{\mathrm{s}}(\delta N) \equiv \sigma\left\{\frac{\delta \varepsilon_{i}(\delta N)-\left\langle\delta \varepsilon_{i}(\delta N)\right\rangle}{\Delta}\right\}
$$

where $\left\langle\delta \varepsilon_{i}(\delta N)\right\rangle$ denotes a linear fit of $\delta \varepsilon_{i}(\delta N)$ as a function of $i$, and $\Delta$ is the mean level spacing. The gate effect is similarly characterized as

$$
\sigma_{\mathrm{g}}\left(\delta U_{p}\right) \equiv \sigma\left\{\frac{\delta \varepsilon_{i}\left(\delta U_{p}\right)-\left\langle\delta \varepsilon_{i}\left(\delta U_{p}\right)\right\rangle}{\Delta}\right\}
$$

with $\delta \varepsilon_{i}\left(\delta U_{p}\right) \equiv \varepsilon_{i}\left(U_{p}^{(0)}+\delta U_{p}\right)-\varepsilon_{i}\left(U_{p}^{(0)}\right)$, and $\left\langle\delta \varepsilon_{i}\left(\delta U_{p}\right)\right\rangle$ its linear fitting. It is more convenient to write $\sigma_{\mathrm{g}}$ as a function of $\delta N^{*} \equiv \delta U_{p} /\left\langle\delta U^{0}\right\rangle$, where $\left\langle\delta U^{0}\right\rangle$ is the average conductance peak spacing. $\delta N^{*}$ can be regarded as the induced electron number due to a change of the gate voltage.

\section{RESULTS AND DISCUSSION}

The scrambling and gate effects are mixed together in CB conductance peak spacings. It is desirable, however, to first study them separately; while the separation of the two effects is difficult to implement experimentally and requires sophisticated design, $\underset{6}{6}$ it is straightforward in numerical investigations. For the scrambling effect, we fix the external confining potential and calculate the TF and KS single-particle spectra at each $N \in[50,70]$. For the gate effect, we fix $N=70$ and $S_{\mathrm{z}}=0$, and scan the plunger gate voltage for $\delta N^{*}$ up to about 20. Statistics are obtained by averaging over different levels and 24 confining gate shapes.

Fig. 3 a) shows the scrambling effect calculated from both TF and KS single-particle spectra. A remarkable feature is
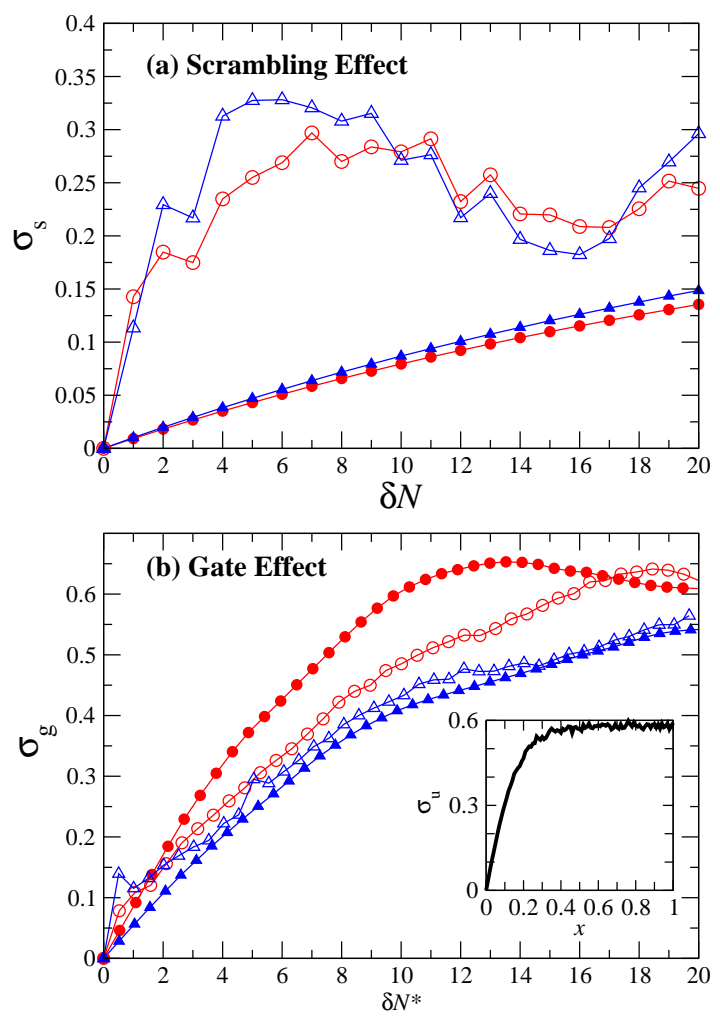

FIG. 3: (Color online) Scrambling magnitude as a function of $\delta N$ (upper) and gate fluctuation as a function of $\delta N^{*}$ (lower) from the TF (filled) or KS (empty) single particle spectra with $z_{\mathrm{d}}=15 \mathrm{~nm}$ (circles) or $50 \mathrm{~nm}$ (triangles). Inset: The result of a universal random matrix model.

the large difference between TF and KS results. $\sigma_{\mathrm{s}}$ from TF spectra increases smoothly as a function of $\delta N$, but is smaller than 0.15 even at $\delta N=20{ }^{29}$ In contrast, $\sigma_{\mathrm{s}}$ from KS spectra increases rapidly at first, and saturates for $\delta N>4$. For $\delta N>$ $8, \sigma_{\mathrm{s}}$ shows some modulations, the reason for which is not yet clear. The effect of the dot depth, $z_{\mathrm{d}}=15 \mathrm{~nm}$ vs. $50 \mathrm{~nm}$, is quite small. The fact that the KS results are much larger than $T F$ ones shows that the residual interaction effects included in the self-consistent $K S$ energies [Eq. (3)] dominate the genuine scrambling evaluated with TF.

Fig. 3 b) shows the gate effect calculated from both TF and KS single-particle spectra. The gate effect in TF and KS spectra are qualitatively the same; in both cases $\sigma_{\mathrm{g}}$ first increases and then saturates at some large $\delta N^{*}$ (about 10 for $z_{\mathrm{d}}=15$ $\mathrm{nm}$ in the TF case). The saturated values of $\sigma_{\mathrm{g}}$ are about 0.6 , larger than that of $\sigma_{\mathrm{s}}(\mathrm{KS})$. The gate effect is quite sensitive to the depth of the dot, especially for the TF case; it is larger for shallow dots, as expected because the gate becomes sharper and better defined. Finally, note that the gate effect is about one order of magnitude larger than the TF scrambling effect.

To further understand these results, we model the parametric evolution of the single-particle spectrum by using a random matrix Gaussian process, 30

$$
H(x)=\cos (x \pi / 2) H_{1}+\sin (x \pi / 2) H_{2}
$$

where $H_{1}$ and $H_{2}$ are independent random matrices belong- 
ing to the Gaussian orthogonal ensemble (GOE) ${ }^{2,28}$ We define $\sigma_{\mathrm{u}}(x)$ similarly to Eqs. (8) or 9) to characterize the change in the single-particle spectrum of $H(x)$ due to the variation of $x$. The inset of Fig. 3 b) shows $\sigma_{\mathrm{u}}$ vs. $x$ obtained from 500 realizations of $30 \times 30$ random matrices. $\sigma_{u}$ saturates at about $x=0.4$, and the saturated value is about 0.6 in agreement with that of $\sigma_{\mathrm{g}}$. We notice that the saturation behavior of $\sigma_{\mathrm{s}}$ from $\mathrm{KS}$ levels is very different from that of $\sigma_{\mathrm{u}}$; this is because the KS single-particle levels contain implicitly some interaction effects so that their variation with $N$ is not a simple Gaussian process.

The agreement between the KS and TF results for the gate effect in Fig. 3 b) is particularly striking when compared to the sharply different magnitudes for the scrambling effect in Fig. 3. a). The underlying reason for this difference between the gate and scrambling effects is the extra electron added in the case of scrambling: First, the contribution to the fluctuations of $\varepsilon_{i, \sigma}^{\mathrm{KS}}$ by most of the residual interactions is small because of the small change in wave functions caused by the additional potential. Second, the contribution by residual interactions involving the added electron $j$ are, however, much larger: the fluctuations here involve the deviation of $\left|\psi_{j}^{\sigma}(\mathbf{r})\right|^{2}$ from the smooth density rather than the small change in an already filled level. Thus the fluctuation of the residual interaction contribution to $\varepsilon_{i, \sigma}^{\mathrm{KS}}$ is substantially stronger in the case of scrambling than for the gate effect.

We now turn to actual spacings between $\mathrm{CB}$ conductance peaks and calculate the scrambling and gate effects. The position for the $N^{\text {th }}$ peak, $U_{g}^{(N)}$, at which the energies for $N-1$ and $N$ electrons are equal, is determined by $\mu\left(N, U_{g}\right) \equiv$ $E_{\mathrm{KS}}\left(N, U_{g}\right)-E_{\mathrm{KS}}\left(N-1, U_{g}\right)=0$ if chemical potentials in the leads are taken to be zero. From the TF spectrum at this $U_{g}$, the scrambling and gate effects are the standard deviation of $\varepsilon_{i}\left(N, U_{g}^{(N)}\right)-\varepsilon_{i}\left(N-1, U_{g}^{(N)}\right)$ and $\varepsilon_{i}\left(N, U_{g}^{(N+1)}\right)-$ $\varepsilon_{i}\left(N, U_{g}^{(N)}\right)$, respectively. For $z_{\mathrm{d}}=15 \mathrm{~nm}$, the former is equal to $0.009 \Delta$, and the latter $0.07 \Delta$, using the same confining gate shapes and parameter ranges as above. Note the good agreement with the results in Fig. 3 for $\delta N=1$ or $\delta N^{*}=1$.

Comparison with earlier evaluations of these effects yields important insights. We start with the scrambling effect; in particular, using the expressions derived in Refs. 1314 for scrambling associated with the Thomas-Fermi spectrum which we think of as the "genuine"scrambling effect - yields $\sigma_{s}^{\text {pred }}(\delta N=1) \simeq 0.06$ for a dot with $N=70$ electrons. This prediction is six times larger than the value obtained here. There are two main differences between the earlier situation and ours: the confining potential here is smooth while it was assumed to be hard-wall in Refs. 13 14, and we effectively have a top gate across the whole dot because of our boundary condition. The insensitivity of the scrambling magnitude to the spacing $z_{d}$ suggests that the top gate has little effect. We therefore conclude that there is significantly less scrambling in a smooth confining potential than in a hard wall dot.

Further support for this conclusion comes from the change in potential upon adding an electron: the hard-wall gives rise to a square-root singularity in this quantity; ${ }^{11}$ the absence of a singularity in the smooth case naturally leads to a weaker effect. As a consequence, the scrambling in the KS spectra, which contains both genuine scrambling and some residual interaction fluctuations [see Eq. (3)], appears to be entirely dominated by the latter. Evaluating the magnitude of these residual interaction fluctuations as an extra electron is added, following the semiclassical random plane wave approach in Refs. 1314 , gives $0.18 \Delta$ for $N=70$. Taking into account that one is not very far into the semiclassical regime, this is quite compatible with the value $\sigma_{s}\left(\delta N^{*}=1\right) \approx 0.13$ computed for the Kohn-Sham spectra [Fig. 3]a)].

For diffusive transport in a weak disordered potential, scrambling has been studied using the statistics of singleparticle wavefunctions in that case.$^{2}$ In that context, scrambling grows linearly with $\delta N$ while residual interaction effects grow as $(\delta N)^{1 / 2}$. It is interesting to note that our data in Fig. 3. a) for a ballistic dot show roughly the same behavior.

With regard to the gate effect, it was argued that a "generic" gate should have the same effect as TF scrambling 14 . For the gate considered here, we however observe a significant difference. A "generic" gate is one which couples approximately uniformly to the dot - a back gate, for instance. The opposite extreme is a gate coupled very locally to a point in the dot, thus producing a rank one perturbation. Such perturbations are known to completely decorrelate the spectra for the phase shift $\pi / 2$ necessary to add an extra electron. ${ }^{31}$ Here we see that lateral plunger gates are an intermediate case: they produce fluctuations which are significantly stronger than the scrambling effect but remain moderate on the scale of the mean level spacing.

The gate effect here is nevertheless strong enough to produce spin transitions. Recently Kogan, et al. reported experimentally a singlet-triplet transition in zero magnetic field driven by changing the confining potential. ${ }^{32}$ In our calculations, we also observe spin transitions caused by gate-induced shape deformation. The probability of a transition - the fraction of peak spacings in which the ground state spin flips as $U_{\mathrm{p}}$ changes - is $5.8 \%$ for $z_{\mathrm{d}}=15 \mathrm{~nm}$ and $4.6 \%$ for $z_{\mathrm{d}}=$ $50 \mathrm{~nm}$. In addition to the simple singlet-triplet transition, different patterns appeared in our calculations. In many cases, the spin flips are paired: in a single spacing interval, the spin changes from one value to another and then back again, which agrees with the general picture connecting spin transitions to avoided-crossings caused by shape-deformation. ${ }^{33}$ In some rare cases, triple-transitions are observed, such as $S_{\mathrm{z}}=2 \rightarrow 1$ $\rightarrow 0 \rightarrow 1$ or $3 / 2 \rightarrow 1 / 2 \rightarrow 3 / 2 \rightarrow 1 / 2$. In other cases, the spin transition is unpaired, presumably because pairing due to an avoided-crossing is destroyed by a change in electron number.

Several experiments have traced the correlation between ground states or excited states as the number of electrons changes, $, 5,8,10$ and often see a surprising degree of correlation. The relatively weak scrambling and gate effects that we find offer a way to understand these results.

To conclude, we have investigated the scrambling and gate effects for two different one-particle spectra - self-consistent Kohn-Sham and Thomas-Fermi - of a realistic model quantum dot. Our main findings are: (1) The genuine scrambling effect - the one associated with the Thomas-Fermi spectra - is significantly smaller for the smooth potential considered here 
than in earlier work using hard wall confinement. (2) As a consequence, scrambling for the Kohn-Sham spectra, which involves both genuine scrambling and residual interactions, is entirely dominated by residual interactions. Its magnitude agrees with those from a random plane wave model of the wave functions. (3) Finally, fluctuations caused by the gate are similar in magnitude for the two spectra. A lateral plunger gate causes significantly larger fluctuations than those caused by scrambling, contrary to the case of a uniform "generic" gate. The magnitude of the gate effect remains nevertheless moderate compared to the mean level spacing.

\section{Acknowledgments}

We thank G. Usaj for valuable comments. This work was supported in part by NSF Grant No. DMR-0103003.
* Current address: Institut für Theoretische Physik, J.W.GoetheUniversität, Frankfurt am Main, Germany

$\dagger$ Permanent address: Laboratoire de Physique Théorique et Modèles Statistiques (LPTMS), 91405 Orsay Cedex, France

${ }^{1}$ L. P. Kouwenhoven, C. M. Marcus, P. L. McEuen, S. Tarucha, R. M. Westervelt, and N. S. Wingreen, in Mesoscopic Electron Transport, edited by L. L. Sohn, G. Schön, and L. P. Kouwenhoven (Kluwer, Dordrecht, 1997), pp. 105-214.

${ }^{2}$ Y. Alhassid, Rev. Mod. Phys. 72, 895 (2000), and references therein.

3 I. L. Aleiner, P. W. Brouwer, and L. I. Glazman, Phys. Rep. 358, 309 (2002), and references therein.

${ }^{4}$ U. Sivan, R. Berkovits, Y. Aloni, O. Prus, A. Auerbach, and G. Ben-Yoseph, Phys. Rev. Lett. 77, 1123 (1996).

5 D. R. Stewart, D. Sprinzak, C. M. Marcus, C. I. Duruöz, and J. S. Harris, Science 278, 1784 (1997).

${ }^{6}$ S. R. Patel, S. M. Cronenwett, D. R. Stewart, A. G. Huibers, C. M. Marcus, C. I. Duruöz, J. S. Harris, K. Campman, and A. C. Gossard, Phys. Rev. Lett. 80, 4522 (1998).

7 S. M. Maurer, S. R. Patel, C. M. Marcus, C. I. Duruöz, and J. S. Harris, Phys. Rev. Lett. 83, 1403 (1999).

${ }^{8}$ S. Lüscher, T. Heinzel, K. Ensslin, W. Wegscheider, and M. Bichler, Phys. Rev. Lett. 86, 2118 (2001).

9 S. Lindemann, T. Ihn, T. Heinzel, W. Zwerger, K. Ensslin, K. Maranowski, and A. Gossard, Phys. Rev. B 66, 195314 (2002).

${ }^{10}$ S. R. Patel, D. R. Stewart, C. M. Marcus, M. Gökcedag, Y. Alhassid, A. D. Stone, C. I. Duruöz, and J. S. Harris, Phys. Rev. Lett. 81, 5900 (1998).

11 Y. M. Blanter, A. D. Mirlin, and B. A. Muzykantskii, Phys. Rev. Lett. 78, 2449 (1997).

12 R. O. Vallejos, C. H. Lewenkopf, and E. R. Mucciolo, Phys. Rev. Lett. 81, 677 (1998).

13 D. Ullmo and H. U. Baranger, Phys. Rev. B 64, 245324 (2001).

${ }^{14}$ G. Usaj and H. U. Baranger, Phys. Rev. B 66, 155333 (2002).

15 A. Szabo and N. S. Ostlund, Modern quantum chemistry (McGraw-Hill, New York, 1989).

16 R. G. Parr and W. Yang, Density-Functional Theory of Atoms and Molecules (Oxford University Press, New York, 1989).

17 D. Ullmo, T. Nagano, S. Tomsovic, and H. U. Baranger, Phys. Rev. B 63, 125339 (2001).

18 D. Ullmo, H. Jiang, W. Yang, and H. U. Baranger, Phys. Rev. B 70, 205309 (2004).

19 A. Messiah, Quantum Mechanics (Wiley, New York, 1958), p. 780.

${ }^{20}$ C.-O. Almbladh and U. von Barth, Phys. Rev. B 31, 3231 (1985).

${ }^{21}$ H. Jiang, H. U. Baranger, and W. Yang, Phys. Rev. B 68, 165337
(2003).

${ }^{22}$ H. Jiang and W. Yang, J. Chem. Phys. 121, 2030 (2004).

${ }^{23}$ H. Jiang, D. Ullmo, W. Yang, and H. U. Baranger, Phys. Rev. B 69, 235326 (2004).

24 J. A. Nixon and J. H. Davies, Phys. Rev. B 41, 7929 (1990).

25 J. H. Davies and I. A. Larkin, Phys. Rev. B 49, 4800 (1994).

26 J. H. Davies, I. A. Larkin, and E. V. Sukhorukov, J. Appl. Phys. 77, 4504 (1995).

27 The underlying physics of the pinned-level model is to assume that electrons can freely move between GaAs surface states and the $2 \mathrm{D}$ electron gas, so that the chemical potential is pinned by the surface states in exposed regions. Indeed the validity of the pinned-level model, though well established for GaAs/AlGaAs devices in equilibrium at finite temperature, is still an open question for lateral quantum dots at near-zero temperature (see, for example, recent experimental studies on this issue in Ref. 34). Here it is worth mentioning that another approach for realistic modeling of quantum dots is to assume the same boundary conditions in a finite volume instead of at infinity as we did (see, for example, Ref. 35). Since the top confining metal gates are usually on the scale of microns, much larger than the quantum dot itself, the difference between these two treatments should be minor. This is actually supported by the fact that, as stated in Sec. IV the effect of the dot depth, $z_{\mathrm{d}}=15 \mathrm{~nm}$ compared with $50 \mathrm{~nm}$, is quite small. With the assumed boundary condition, the image term in the Hartree potential arises naturally by taking advantage of the linearity of Poisson equation.

${ }^{28}$ O. Bohigas, in Chaos and Quantum Physics, edited by M. J. Giannoni, A. Voros, and J. Jinn-Justin (North-Holland, Amsterdam, 1990), pp. 87-199.

29 In contributing to the conductance peak spacing, the scrambling effect contains two parts $:^{13}$ the contribution from the top level the quantity discussed here - and that from the sum over all other occupied levels. In our numerical calculations, we confirm the expectation that the second term is small compared to the first.

30 Y. Alhassid and S. Malhotra, Phys. Rev. B 60, R16315 (1999).

31 I. L. Aleiner and K. A. Matveev, Phys. Rev. Lett. 80, 814 (1998).

32 A. Kogan, G. Granger, M. A. Kastner, D. Goldhaber-Gordon, and H. Shtrikman, Phys. Rev. B 67, 113309 (2003).

${ }^{33}$ H. U. Baranger, D. Ullmo, and L. I. Glazman, Phys. Rev. B 61, R2425 (2000).

34 A. Kawaharazuka, T. Saku, C. A. Kikuchi, Y. Horikoshi, and Y. Hirayama, Phys. Rev. B 63, 245309 (2001).

35 L.-X. Zhang, P. Matagne, J. P. Leburton, R. Hanson, and L. P. Kouwenhoven, Phys. Rev. B 69, 245301 (2004). 